\title{
The number of periodic points of smooth maps $\dagger$
}

\author{
TAKASHI MATSUOKA \\ Department of Mathematics, Naruto University of Education, Naruto, \\ Tokushima 772, Japan
}

(Received 27 April 1987 and revised 22 February 1988)

\begin{abstract}
Let $f: M \rightarrow M$ be a $C^{1}$ map on a compact manifold. We give a topological condition under which $f$ has an even number of periodic points with a given period. We also obtain a sufficient condition, in terms of homology, for $f$ to have infinitely many periodic points.
\end{abstract}

\section{Introduction}

In [14], Levinson obtained a result concerning the number of periodic points of diffeomorphisms of a disk. He gave a classification of periodic points and proved certain equalities between the cardinal numbers of the classes of periodic points. His equalities were improved by Massera [15] and extended to compact manifolds by Shiraiwa [18]. Recently, Dold obtained an extension of these equalities to smooth maps on manifolds in [5] (Theorem 1 below).

In this paper, we give two applications of the Dold's equalities to the study of periodic points of smooth maps. The first application is concerned with the problem of whether the number $\nu(n)$ of periodic points of a given period $n$ is even or odd. We give a condition on $n$ and on the homotopy class of the map under which $\nu(n)$ is even. This generalizes results in $[14,15,18]$.

In the second application, we generalize some results of Franks [8,9] on the existence of infinitely many periodic points.

In the last section, we generalize the Dold's equalities to isolated sets of periodic points.

\section{The Dold's equalities}

Let $M$ be a $C^{\infty}$ manifold (possibly with boundary), $V$ an open set of $M$, and let $f: V \rightarrow M$ be a continuous map. For positive integers $n$, define the iterates $f^{n}: V_{n} \rightarrow M$ inductively by

$$
f^{1}=f, \quad V_{n}=f^{-1}\left(V_{n-1}\right), \quad f^{n}(x)=f^{n-1}(f(x)) \quad \text { for } n>1 .
$$

Denote by Fix $\left(f^{n}\right)$ the fixed point set of $f^{n}$, i.e. the set of points $x \in V_{n}$ with $f^{n}(x)=x$.

Suppose Fix $\left(f^{n}\right)$ is compact. Then Fix $\left(f^{m}\right)$ is also compact for any divisor $m$ of $n$ because Fix $\left(f^{m}\right)$ is a closed subset of Fix $\left(f^{n}\right)$, and hence the fixed point 
index $I\left(f^{m}\right)$ of $f^{m}$ is defined (cf, [4]). Define an integer $I_{n}(f)$ by

$$
I_{n}(f)=\sum_{\tau \subset P(n)}(-1)^{|\tau|} I\left(f^{n: \tau}\right),
$$

where $P(n)$ is the set of all primes which divide $n$, the sum extends over all subsets $\tau$ of $P(n),|\tau|=$ cardinality of $\tau$, and $n: \tau=n\left(\prod_{p \in \tau} p\right)^{-1}=n$ divided by all $p \in \tau$. For example, if $n$ is a prime number, then $I_{n}(f)=I\left(f^{n}\right)-I(f)$.

Definition 1. We set

$$
\operatorname{Per}(n)=\operatorname{Fix}\left(f^{n}\right)-\bigcup_{m<n} \operatorname{Fix}\left(f^{m}\right),
$$

and call an element of Per $(n)$ an n-periodic point, or simply a periodic point, of $f$.

Now assume that $f$ is a $C^{1}$ map. For $x \in \operatorname{Per}(n)$, let $D f^{n}(x)$ denote the derivative of $f^{n}$ at $x$, and let $a_{+}(x)$ (resp. $a_{-}(x)$ ) be the number of real eigenvalues $\lambda$ of $D f^{n}(x)$ with $\lambda>1$ (resp. $\lambda<-1$ ) (counting multiplicity). We then classify the $n$-periodic points by these numbers $a_{+}(x), a_{-}(x)$; namely, we divide the set $\operatorname{Per}(n)$ into the following four subsets:

$$
\begin{aligned}
& \operatorname{Per}_{\mathrm{EE}}(n)=\left\{x \in \operatorname{Per}(n) \mid a_{+}(x), a_{-}(x) \text { are even }\right\}, \\
& \operatorname{Per}_{\mathrm{EO}}(n)=\left\{x \in \operatorname{Per}(n) \mid a_{+}(x) \text { is even, } a_{-}(x) \text { is odd }\right\}, \\
& \operatorname{Per}_{\mathrm{OE}}(n)=\left\{x \in \operatorname{Per}(n) \mid a_{+}(x) \text { is odd, } a_{-}(x) \text { is even }\right\}, \\
& \operatorname{Per}_{\mathrm{OO}}(n)=\left\{x \in \operatorname{Per}(n) \mid a_{+}(x), a_{-}(x) \text { are odd }\right\}
\end{aligned}
$$

We let

$$
\begin{array}{rlrl}
\nu_{\mathrm{EE}}(n)=\left|\operatorname{Per}_{\mathrm{EE}}(n)\right|, & & \nu_{\mathrm{EO}}(n)=\left|\operatorname{Per}_{\mathrm{EO}}(n)\right|, \\
\nu_{\mathrm{OE}}(n)=\left|\operatorname{Per}_{\mathrm{OE}}(n)\right|, & \nu_{\mathrm{OO}}(n)=\left|\operatorname{Per}_{\mathrm{OO}}(n)\right|, \\
\nu_{\mathrm{E}}(n)=\nu_{\mathrm{EE}}(n)+\nu_{\mathrm{EO}}(n), & \nu_{\mathrm{O}}(n)=\nu_{\mathrm{OE}}(n)+\nu_{\mathrm{OO}}(n),
\end{array}
$$

where | | denotes the cardinality.

Definition 2. A fixed point $x$ of $f^{n}$ is regular if the derivative $D f^{n}(x)$ does not have 1 as an eigenvalue.

Note that if $x \in \operatorname{Per}(n)$ is regular as a fixed point of $f^{n}$ and $x$ is contained in the interior Int $M$ of $M$, then $a_{+}(x)$ determines the fixed point index $I\left(f^{n}, x\right)$ of $x$ for the map $f^{n}$. In fact (cf, [5, p. 431]),

$$
\begin{aligned}
I\left(f^{n}, x\right) & =1 & & \text { if } a_{+}(x) \text { is even, } \\
& =-1 & & \text { if } a_{+}(x) \text { is odd. }
\end{aligned}
$$

Note also that if $x \in \operatorname{Per}(n)$ is regular as a fixed point of $f^{2 n}$ and $x \in \operatorname{Int} M$, then $a_{-}(x)$ determines whether $x$ is inverting in the sense of Dold [5]. (Such a point $x$ is said to be inverting if $I\left(f^{2 n}, x\right)=-I\left(f^{n}, x\right)$.) In fact, it is easy to see that $x$ is inverting if and only if $a_{-}(x)$ is odd (cf, [5]).

We now fix a positive integer $n$, and assume that every fixed point of $f^{n}$ is regular and contained in Int $M$. In this case, as is easily seen by the relations between the fixed point index and the numbers $a_{+}(x), a_{-}(x)$ mentioned above, the number $\nu_{\mathrm{E}}(n)$ (resp. $\nu_{\mathrm{O}}(n)$ ) is equal to the number of $n$-periodic points $x$ with index $I\left(f^{n}, x\right)=1$ (resp. -1$)$. Moreover, when $n$ is even, the number $\nu_{\mathrm{EO}}(n / 2)\left(\right.$ resp. $\left.\nu_{\mathrm{OO}}(n / 2)\right)$ is 
equal to the number of inverting (n/2)-periodic points $x$ with index $I\left(f^{n / 2}, x\right)=1$ (resp. -1 ). The following result of Dold [5, Proposition (6.2)] gives a relation between these numbers and the number $I_{n}(f)$ :

THEOREM 1 (Dold). Let $f: V \rightarrow M$ be a $C^{1}$ map defined on an open subset $V$ of $a$ manifold $M$, and let $n$ be a positive integer. Suppose that $f^{n}$ has only finitely many fixed points and that every fixed point of $f^{n}$ is regular and contained in the interior Int $M$ of $M$. Then

$$
\begin{aligned}
I_{n}(f) & =\nu_{\mathrm{E}}(n)-\nu_{\mathrm{O}}(n) & & \text { if } n \text { is odd, } \\
& =\nu_{\mathrm{E}}(n)-\nu_{\mathrm{O}}(n)-2\left(\nu_{\mathrm{EO}}(n / 2)-\nu_{\mathrm{OO}}(n / 2)\right) & & \text { if } n \text { is even. }
\end{aligned}
$$

In some special cases, this theorem was proved and was applied to the study of periodic solutions of periodic differential systems by Levinson [14, §6], Massera [15], Shiraiwa [18]. In [5] Dold used this theorem to prove that $n$ divides the number $I_{n}(f)$ for any continuous map $f$ defined on an open set of an ENR with Fix $\left(f^{n}\right)$ compact. (This result was also obtained by Zabreiko and Krasnosel'skii [19], [13, Theorem 31.4].) In $\S \S 3$ and 4, we shall give two other applications of Theorem 1.

\section{The first application}

Throughout this section, we assume that $M$ is a compact manifold.

Let $f: M \rightarrow M$ be a continuous map.

Definition 3. A positive integer $k$ is an $L_{2}$-period of $f$ if

$$
L_{2}\left(f^{i+k}\right)=L_{2}\left(f^{i}\right) \quad \text { for any } i \geq 1,
$$

where $L_{2}\left(f^{i}\right) \in \mathbb{Z}_{2}$ denotes the $\bmod 2$ reduction of the Lefschetz number $L\left(f^{i}\right)$ of $f^{i}$, i.e. $L_{2}\left(f^{i}\right)=0$ or 1 according as $L\left(f^{i}\right)$ is even or odd. We denote the minimal $L_{2}$-period of $f$ by $\alpha(f)$.

For example, let $f: S^{1} \rightarrow S^{1}$ be a continuous map on the circle. Then $L\left(f^{i}\right)=1-d^{i}$, where $d$ is the degree of $f$, and hence $L_{2}\left(f^{i}\right)=0$ for all $i$ or $L_{2}\left(f^{i}\right)=1$ for all $i$. Therefore, in this case, $\alpha(f)=1$.

The following proposition clearly implies that $\alpha(f)$ always exists and is an odd number:

Proposition 1. Let $p$ be a prime number and $A$ a square matrix with entries in $\mathbb{Z} / p \mathbb{Z}$. Then there exists a positive integer $k$ such that for any $i \geq 1$,

$$
\operatorname{tr} A^{i+k}=\operatorname{tr} A^{i}
$$

and that $k$ is not divisible by $p$.

Proof. Since $\left\{A^{i} \mid i \geq 1\right\}$ is a finite set, there exist positive integers $I$ and $l$ such that $A^{I}=A^{I+l}$. Then for any $i \geq I$,

$$
A^{i}=A^{I} A^{i-I}=A^{I+l} A^{i-I}=A^{i+l} \text {. }
$$

Let $i$ be a positive integer. Then, $i q \geq I$ for some power $q$ of $p$. Since

$$
\operatorname{tr} B^{p^{\prime}}=\operatorname{tr} B
$$

for any $j \geq 0$ and any square matrix $B$ with entries in $\mathbb{Z} / p \mathbb{Z}$ ([2, Proposition 5], 
$[17$, Lemma]), we have

$$
\operatorname{tr} A^{i}=\operatorname{tr} A^{i q}=\operatorname{tr} A^{i q+l q}=\operatorname{tr} A^{(i+l) q}=\operatorname{tr} A^{i+l}
$$

Hence $l$ is a period for $\left\{\operatorname{tr} A^{i}\right\}_{i \geq 1}$. Decompose $l$ as $k r$, where $k$ is not divisible by $p$ and $r$ is a power of $p$. Then, by (3.1), for any $i$,

$$
\operatorname{tr} A^{i+k}=\operatorname{tr} A^{(i+k) r}=\operatorname{tr} A^{i r}=\operatorname{tr} A^{i}
$$

Thus $k$ is also a period, and the proof is completed.

Given an $n$-periodic point $x$ of $f$, we call the set $\left\{f^{i}(x) \mid i \geq 1\right\}$ an n-periodic orbit of $f$. As an application of Theorem 1, we have:

THEOREM 2. Let $f: M \rightarrow M$ be a $C^{1}$ map and $n$ an odd number with $n \geq 3$. Suppose that every fixed point of $f^{n}$ is regular and contained in Int $M$. Suppose also that $n$ is divisible by $\alpha(f)^{2}$ or by a prime number $p$ which is congruent to $2^{i}$ modulo $\alpha(f)$ for some $i \geq 0$. Then, the number $\bar{\nu}(n)$ of $n$-periodic orbits of $f$ is even.

Proof. By the assumption, $\mid$ Fix $\left(f^{n}\right) \mid<\infty$. Hence by Theorem 1,

$$
\bar{\nu}(n)=|\operatorname{Per}(n)| / n=2 \nu_{0}(n) / n+I_{n}(f) / n \text {. }
$$

Hence, it is sufficient for the proof to show that the $\bmod 2$ reduction $I_{n, 2}(f)$ of $I_{n}(f)$ is equal to zero. Let $\alpha=\alpha(f)$. Suppose first $\alpha^{2} \mid n$. Then for any $\tau \subset P(n), n: \tau$ is a multiple of $\alpha$. Therefore, since $L_{2}\left(f^{k}\right)=L_{2}\left(f^{\alpha}\right)$ for any multiple $k$ of $\alpha$,

$$
I_{n, 2}(f)=\left(\sum_{\tau \subset P(n)}(-1)^{|\tau|}\right) L_{2}\left(f^{\alpha}\right)=0 .
$$

Next, suppose $n$ is divisible by a prime $p$ with $p \equiv 2^{i}(\bmod \alpha)$ for some $i$. Decompose $n$ as $n=p^{j} r$, where $j, r \geq 1$ and $r$ is not divisible by $p$. Then

$$
I_{n}(f)=I_{r}\left(f^{p^{j}}\right)-I_{r}\left(f^{p^{j-1}}\right) .
$$

Since $\alpha$ is a period for the sequence $\left\{I_{r, 2}\left(f^{i}\right)\right\}_{i \geq 1}$,

$$
I_{r, 2}\left(f^{p^{s}}\right)=I_{r, 2}\left(f^{2^{i s}}\right) \text { for } s \geq 0 .
$$

Hence by (3.1), $I_{r, 2}\left(f^{p^{s}}\right)=I_{r, 2}(f)$ for $s \geq 0$.

Therefore by $(3.2), I_{n, 2}(f)=I_{r, 2}(f)-I_{r, 2}(f)=0$.

Let $b(M)$ be the maximum of the Betti numbers of $M$. It is easy to show that if $b(M)=1$ (resp. 2) then $\alpha(f)=1$ (resp. 1 or 3). Hence, by Theorem 2, we have:

Corollary. Let $n$ be an odd number. Assume either that $b(M)=1, n \geq 3$, or that $b(M)=2, n \geq 5$. Let $f: M \rightarrow M$ be $a C^{1}$ map and suppose that every fixed point of $f^{n}$ is regular and contained in Int $M$. Then $\bar{\nu}(n)$ is even.

In the case where $f$ is a diffeomorphism of a disk, this corollary has been obtained by Levinson [14], Massera [15]. Theorem 2 follows from Shiraiwa [18, Theorem 3] in the case where $f$ is a diffeomorphism with $L(f)=L\left(f^{i}\right)$ for any $i$.

When $b(M) \geq 3$, the condition for $\bar{\nu}(n)$ to be even does not seem simple in many cases. For example, let $M$ be a disk with 3 holes. Suppose a $3 \times 3$ matrix $\left(a_{i j}\right)$ representing the homomorphism $f_{*}: H_{1}(M ; \mathbb{Z}) \rightarrow H_{1}(M ; \mathbb{Z})$ is given by $a_{i j}=0$ or 1 according as $i+j \leq 3$ or $i+j>3$ respectively. Then $\alpha(f)=7$ and the computation 
of $I_{n}(f)$ shows that, for $n$ odd, $\bar{\nu}(n)$ is odd if and only if $n=r^{i}$ or $7 r^{i}$, where $r$ is a prime number satisfying $r \equiv 3,5,6(\bmod 7)$.

\section{The existence of infinitely many periodic points}

In this section, we shall generalize some theorems of Franks in [8,9] on the existence of infinitely many periodic points. Here we assume the following conditions:

(i) $M$ is a compact manifold and $f: M \rightarrow M$ is a $C^{1}$ map.

(ii) $f^{n}$ has only regular fixed points for any $n \geq 1$, and $f$ has no periodic points on the boundary of $M$.

(iii) All non-zero eigenvalues of $f_{*}: H_{*}(M ; \mathbb{Q}) \rightarrow H_{*}(M ; \mathbb{Q})$ are $q$ th roots of unity for some $q>0$.

We first define homotopy invariant integers $\gamma(f, a)$ for positive integers $a$, which play a central role in the generalization of the Franks's results. For a positive integer $r$ and $i \geq 0$, let $\gamma(r, i)$ be the number of eigenvalues $\lambda$ of $f_{* i}: H_{i}(M ; \mathbb{Q}) \rightarrow H_{i}(M ; \mathbb{Q})$ which are $r$ th primitive roots of unity (counting multiplicity) and set

$$
\gamma_{r}(f)=\sum_{i \geq 0}(-1)^{i} \gamma(r, i) / \phi(r),
$$

where $\phi(r)$ denotes the Euler function, i.e. $\phi(r)$ is the number of integers $s$ such that $1 \leq s \leq r$ and $(r, s)$, the greatest common divisor of $r$ and $s$, is equal to 1 . For a positive integer $a$, let

$$
\gamma(f, a)=\sum_{r} \mu(r) \gamma_{a r}(f),
$$

where the sum is taken over all odd numbers $r$, and $\mu(r)$ is the Möbius function, i.e. $\mu(1)=1, \mu(r)=(-1)^{k}$ if $r$ is a product of $k$ distinct primes, and $\mu(r)=0$ otherwise. (This definition of $\gamma(f, a)$ makes sense, because $\gamma_{a r}(f)=0$ if $a r+q$.) For example, if $q=2^{i} 3, i \geq 0$, then $\gamma(f, 1)=\gamma_{1}(f)-\gamma_{3}(f), \gamma(f, 3)=\gamma_{3}(f)$, and $\gamma(f, a)=0$ for odd $a \geq 5$.

Now let

$$
\bar{\nu}_{a b}(n)=\nu_{a b}(n) / n \text { for } a, b=\mathrm{E}, \mathrm{O} .
$$

Then these are clearly non-negative integers and we have:

THEOREM 3. Let $a$ be an odd number and let $i(a)$ denote the smallest $i \geq 0$ such that $2^{i} a$ does not divide $q$. Then for any $i \geq i(a)-1$, we have

$$
\bar{\nu}_{\mathrm{EO}}\left(2^{i} a\right)-\bar{\nu}_{\mathrm{OO}}\left(2^{i} a\right)=\gamma(f, a)-\sum_{j=0}^{i}\left(\bar{\nu}_{\mathrm{EE}}\left(2^{j} a\right)-\bar{\nu}_{\mathrm{OE}}\left(2^{j} a\right)\right) .
$$

Recall that an $n$-periodic point $x$ is said to be inverting if $I\left(f^{2 n}, x\right)=-I\left(f^{n}, x\right)$ $(\$ 2)$. Then it is clear that $x$ is an inverting $n$-periodic point if and only if $x \in$ $\operatorname{Per}_{\text {EO }}(n) \cup \operatorname{Per}_{\text {Oo }}(n)$. Theorem 3 immediately implies the following corollaries concerning the existence of infinitely many inverting periodic points:

COROLlary 1. Let $a$ be an odd number. Suppose that

$$
\gamma(f, a)>\sum_{j=0}^{\infty} \bar{\nu}_{\mathrm{EE}}\left(2^{j} a\right) \quad \text { or } \gamma(f, a)<-\sum_{j=0}^{\infty} \bar{\nu}_{\mathrm{OE}}\left(2^{j} a\right)
$$


Then for any $i \geq i(a)-1, f$ has an inverting $2^{i} a$-periodic point.

COROLlary 2. Let $a$ be an odd number satisfying $\gamma(f, a)=0$. Suppose either

(i) $\nu_{\mathrm{EE}}\left(2^{i} a\right)=\nu_{\mathrm{OO}}\left(2^{i} a\right)=0$ for any $i \geq 0$, or

(ii) $\nu_{\mathrm{EO}}\left(2^{i} a\right)=\nu_{\mathrm{OE}}\left(2^{i} a\right)=0$ for any $i \geq 0$.

Then we have

$$
\bar{\nu}_{\mathrm{EO}}\left(2^{i} a\right)+\bar{\nu}_{\mathrm{OO}}\left(2^{i} a\right)=\sum_{j=0}^{i}\left(\bar{\nu}_{\mathrm{EE}}\left(2^{j} a\right)+\bar{\nu}_{\mathrm{OE}}\left(2^{j} a\right)\right)
$$

for any $i \geq i(a)-1$. In particular, if $f$ has a $2^{i_{0}}$ a-periodic point for some $i_{0} \geq i(a)-1$, then for each $k \geq i_{0}, f$ has an inverting $2^{k}$ a-periodic point.

Corollary 1 generalizes the result of Franks [8]. In [8], Corollary 1 has been proved in the special case where $a=1, M$ is a circle, a sphere, a closed interval, or a disk, under some additional assumptions. Also, Corollary 2 generalizes Theorem $\mathrm{C}$ in Franks [9]; in this theorem, Corollary 2 has been obtained in the restricted case of $i(a)=0$. (Note that $i(a)=0$ implies $\gamma(f, a)=0$, and that the notion of an inverting periodic point is identical with that of a 'twisted' periodic point in [9].) Thus Theorem 3 generalizes and unifies results of Franks in $[8,9]$, which were obtained by using the Lefschetz zeta function.

Proof of Theorem 3. Using Theorem 1, we can prove the following equalities by induction on $i$ :

$$
\begin{aligned}
& \bar{\nu}_{\mathrm{EO}}\left(2^{i} a\right)-\bar{\nu}_{\mathrm{OO}}\left(2^{i} a\right) \\
& =\sum_{j=0}^{i}\left(\bar{\nu}_{\mathrm{OE}}\left(2^{j} a\right)-\bar{\nu}_{\mathrm{EE}}\left(2^{j} a\right)+\bar{I}_{2^{j} a}(f)\right),
\end{aligned}
$$

where $\bar{I}_{k}(f)=I_{k}(f) / k$. Hence it is sufficient to show that if $i \geq i(a)-1$, then

$$
\sum_{j=0}^{i} \bar{I}_{2^{j} a}(f)=\gamma(f, a) .
$$

For $r \geq 1$, let $A_{r}$ be the set of all $r$ th primitive roots of unity. Let

$$
\begin{gathered}
\psi(r, j)=\sum_{\lambda \in A_{r}} \lambda^{j} \quad \text { for } j \geq 1, \\
M_{r}(n)=\frac{1}{n}\left(\sum_{\tau \subset P(n)}(-1)^{|\tau|} \psi(r, n: \tau)\right) .
\end{gathered}
$$

Then clearly we have:

$$
\bar{I}_{n}(f)=\sum_{r \mid q} \gamma_{r}(f) M_{r}(n)
$$

It is easy to show that

$$
M_{r}(n)=\frac{1}{n} \sum_{d \mid n} \mu(d) \psi(r, n / d) .
$$

LEMMA. For $n, r \geq 1, M_{r}(n)=\mu(r / n)$, where $\mu(r / n)$ means 0 if $r$ is not divisible by $n$.

Proof. We prove this lemma by induction on the number $s(n)$ of primes dividing $n$. If $s(n)=0$, then $n=1$ and the lemma is trivial. Assume the lemma holds for any $r$ and any $n$ such that $s(n)$ is less than an integer $s$. Let $r, n \geq 1$ and suppose $s(n)=s$. 
Decompose $n$ and $r$ as $n=p^{i} n^{\prime}, r=p^{j} r^{\prime}$, where $n^{\prime}, r^{\prime}, i \geq 1, j \geq 0$, and $p$ is a prime which does not divide $n^{\prime}, r^{\prime}$. Let $v=\min \{i, j\}$ and $w=\min \{i-1, j\}$. For any $k$, we have

$$
\psi(r, k n)=\psi\left(r^{\prime \prime}, n\right) \phi(r) / \phi\left(r^{\prime \prime}\right),
$$

where $r^{\prime \prime}=r /(r, k)$, because the $k$ times power of an $r$ th primitive root of unity is an $r /(r, k)$ th primitive root of unity. Hence, by the induction hypothesis, we have

$$
\begin{aligned}
M_{r}(n) & =\frac{1}{n} \sum_{d^{\prime} \mid n^{\prime}} \mu\left(d^{\prime}\right)\left\{\psi\left(r, p^{i} n^{\prime} / d^{\prime}\right)-\psi\left(r, p^{i-1} n^{\prime} / d^{\prime}\right)\right\} \\
& =\frac{n^{\prime}}{n}\left\{M_{r p^{-v}}\left(n^{\prime}\right) \phi_{v}-M_{r p^{-w}}\left(n^{\prime}\right) \phi_{w}\right\} \\
& =p^{-i} \mu\left(r^{\prime} / n^{\prime}\right)\left\{\mu\left(p^{j-v}\right) \phi_{v}-\mu\left(p^{j-w}\right) \phi_{w}\right\}
\end{aligned}
$$

where $\phi_{v}=\phi\left(p^{j}\right) / \phi\left(p^{j-v}\right), \phi_{w}=\phi\left(p^{j}\right) / \phi\left(p^{j-w}\right)$.

If $\boldsymbol{n}$ does not divide $r$, then we can assume $p^{i}$ does not divide $r$. Therefore, $v=w$ and by (4.3) $M_{r}(n)=0$. If $n$ divides $r$, then $v=i$ and $w=i-1$. Hence by (4.3), $M_{r}(n)=\mu(r / n)$. Thus the proof is completed.

Now let $i \geq i(a)-1$. By (4.2) and Lemma, since

$$
\sum_{j=0}^{i} \mu\left(r / 2^{j} a\right)=0 \quad \text { for } r \text { even, }
$$

we have

$$
\begin{aligned}
\sum_{j=0}^{i} \bar{I}_{2^{j} a}(f) & =\sum_{r \mid q} \gamma_{r}(f) \sum_{j=0}^{i} \mu\left(r / 2^{j} a\right) \\
& =\sum_{r \text { odd }} \gamma_{r}(f) \mu(r / a)=\gamma(f, a) .
\end{aligned}
$$

Thus the proof is completed.

\section{An extension of Theorem 1}

Let $M$ be a $C^{\infty}$ manifold, $V$ an open set of $M$, and let $f: V \rightarrow M$ be a continuous map. Let $n$ be a positive integer, and $S$ an isolated set of fixed points of $f^{n}$,i.e. an open and closed subset of Fix $\left(f^{n}\right)$. If $S$ is compact, then an integer $I_{n}(f, S)$ is defined by

$$
I_{n}(f, S)=\sum_{\tau \in P(n)}(-1)^{|\tau|} I\left(f^{n: \tau}, S_{\tau}\right)
$$

where $S_{\tau}=$ Fix $\left(f^{n: \tau}\right) \cap S$ and $I\left(f^{n: \tau}, S_{\tau}\right)$ denotes the fixed point index of $S_{\tau}$ for the $\operatorname{map} f^{n: \tau}$. For $a, b=\mathrm{E}, \mathrm{O}$, let

$$
\nu_{a, b}(n, S)=\left|\operatorname{Per}_{a b}(n) \cap S\right|, \nu_{a}(n, S)=\nu_{a \mathrm{E}}(n, S)+\nu_{a \mathrm{O}}(n, S) .
$$

Note that if $S=$ Fix $\left(f^{n}\right)$, then $I_{n}(f, S)=I_{n}(f), \nu_{a b}(n, S)=\nu_{a b}(n)$. We then have the following generalization of Theorem 1 :

THEOREM 4. Let $f: V \rightarrow M$ be a $C^{1}$ map defined on an open subset $V$ of a manifold $M$, and let $n$ be a positive integer. Let $S$ be a finite subset of Fix $\left(f^{n}\right)$. Suppose that 
every fixed point of $f^{n}$ contained in $S$ is regular and that $S \subset \operatorname{Int} M$. Then

$$
\begin{aligned}
I_{n}(f, S) & =\nu_{\mathrm{E}}(n, S)-\nu_{\mathrm{O}}(n, S) & & \text { if } n \text { is odd, } \\
& =\nu_{\mathrm{E}}(n, S)-\nu_{\mathrm{O}}(n, S)-2\left(\nu_{\mathrm{EO}}(n / 2, S)-\nu_{\mathrm{OO}}(n / 2, S)\right) & & \text { if } n \text { is even. }
\end{aligned}
$$

Proof. In the case where $f(S)=S$, the theorem can be considered as a special case of Theorem 1. In fact, if we choose an open neighborhood $W$ of $S$ with $W \cap$ Fix $\left(f^{n}\right)=S$, then Fix $\left(f^{m}\right) \cap S=$ Fix $\left(\left(\left.f\right|_{w}\right)^{m}\right)$ for any divisor $m$ of $n$, and hence $I_{n}(f, S)=I_{n}\left(\left.f\right|_{W}\right)$. Therefore, Theorem 4 is immediately obtained by applying Theorem 1 to the map $\left.f\right|_{w}$.

The general case can be also proved by using Theorem 1 in the following way. Since

$$
I_{n}(f, S)=\sum_{x \in S} I_{n}(f,\{x\}), \quad \nu_{a b}(n, S)=\sum_{x \in S} \nu_{a b}(n,\{x\}),
$$

without loss of generality, we may assume that $S$ consists of only one point $x$. Let $m$ be the least period of $x$ and let $n^{\prime}=n / m$. Then it is easy to see that Fix $\left(f^{n: \tau}\right) \cap\{x\}$ is not empty if and only if $\tau \subset P\left(n^{\prime}\right)$. Hence we have

$$
I_{n}(f,\{x\})=\sum_{\tau \in P\left(n^{\prime}\right)}(-1)^{|\tau|} I\left(f^{n: \tau}, x\right) .
$$

Choose an open neighborhood $W$ of $x$ such that Fix $\left(f^{n}\right) \cap W=\{x\}$ and let $g=\left.f^{m}\right|_{W}$. Then

$$
I\left(f^{n: \tau}, x\right)=I\left(\left(f^{m}\right)^{n^{\prime}: \tau}, x\right)=I\left(g^{n^{\prime}: \tau}\right) .
$$

Therefore by (5.2), $I_{n}(f,\{x\})=I_{n},(g)$. Applying Theorem 1 to $g$ and $n^{\prime}$, we can verify that $I_{n}(g)$ is equal to the right-hand side of the desired equality (in the case of $S=\{x\})$. Hence the proof is completed.

For the application of Theorem 4 to the study of the number of periodic points, one must compute the number $I_{n}(f, S)$. This, however, is difficult in general. But if $I_{n}(f, S)$ is invariant under homotopy in some sense, this invariance will be of great help in the computation. In the following, we give a sufficient condition on $S$ for the number $I_{n}(f, S)$ to be invariant under homotopy by making use of Nielsen fixed point theory. For a general reference on Nielsen fixed point theory, see Jiang [11]. See also Brown [3].

In the rest of this paper, we assume that $V=M$ and $M$ is compact and connected. Let $p: \tilde{M} \rightarrow M$ be the universal covering of $M$. Denote the group of covering transformations of $\tilde{M}$ by $\Gamma$.

Definition 4. We say that two liftings $\tilde{f}$ and $\tilde{f}^{\prime}$ of $f: M \rightarrow M$ are conjugate if there exists an element $\gamma$ of $\Gamma$ such that $\tilde{f}^{\prime}=\gamma \circ \tilde{f} \circ \gamma^{-1}$. The conjugacy class [ $\left.\tilde{f}\right]$ of the lift $\tilde{f}$ is said to be a lifting class of $f$. The subset $p($ Fix $(\tilde{f}))$ of Fix $(f)$ is called the Nielsen class (or the fixed point class) of $f$ determined by the lifting class $[\tilde{f}]$ (see $[11$, p. 5]). (Note that this definition does not depend on the choice of a representative $\tilde{f}$ of a lifting class; indeed, if $[\tilde{f}]=\left[\tilde{f}^{\prime}\right]$, then $p(\operatorname{Fix}(\tilde{f}))=p\left(\operatorname{Fix}\left(\tilde{f}^{\prime}\right)\right)$.)

The following are known (cf, [11]):

(i) A Nielsen class is an isolated set of fixed points of $f$.

(ii) There are only finitely many non-empty Nielsen classes, and Fix $(f)$ splits into a disjoint union of Nielsen classes. 
(iii) Two fixed points $x$ and $y$ of $f$ belong to the same Nielsen class if and only if there exists a path $c$ in $M$ from $x$ to $y$ such that $f \circ c$ is homotopic to $c$ with endpoints fixed.

Note that by (i), $I_{n}(f, N)$ is defined for any Nielsen class $N$ of $f^{n}$, and by (ii),

$$
I_{n}(f)=\sum_{N} I_{n}(f, N) \quad \text { and } \quad \nu_{a b}(n)=\sum_{N} \nu_{a b}(n, N)
$$

where $N$ ranges over the set of all Nielsen classes of $f^{n}$.

Let $g_{0}, g_{1}: M \rightarrow M$ be homotopic continuous maps, and $H: M \times[0,1] \rightarrow M$ a homotopy from $g_{0}$ to $g_{1}$. A homotopy $\tilde{H}: \tilde{M} \times[0,1] \rightarrow \tilde{M}$ is called a lifting of the homotopy $H$ if $\tilde{H}_{t}$ is a lifting of $H_{t}$ for any $t \in[0,1]$, where $H_{t}: M \rightarrow M, \tilde{H}_{t}: \tilde{M} \rightarrow \tilde{M}$ are defined by $H_{t}(x)=H(x, t), \tilde{H}_{t}(\tilde{x})=\tilde{H}(\tilde{x}, t)$.

Definition 5. Let $\tilde{g}_{i}$ be a lifting of $g_{i}, i=0,1$. We say that the lifting class [ $\tilde{g}_{0}$ ] (and the Nielsen class determined by $\left[\tilde{g}_{0}\right]$ ) corresponds to the lifting class $\left[\tilde{g}_{1}\right]$ (and the Nielsen class determined by $\left[\tilde{g}_{1}\right]$ ) via the homotopy $H$ if $H$ has a lifting $\tilde{H}$ from $\tilde{g}_{0}$ to $\tilde{g}_{1}$.

Now let $f_{0}, f_{1}: M \rightarrow M$ be homotopic continuous maps, and $H: M \times[0,1] \rightarrow M$ a homotopy from $f_{0}$ to $f_{1}$. Let $n$ be a positive integer and define a homotopy $H^{n}: M \times[0,1] \rightarrow M$ from $f_{0}^{n}$ to $f_{1}^{n}$ by $H^{n}(x, t)=\left(H_{t}\right)^{n}(x)$. Then we have:

Proposition 2. Let $N_{i}$ be a Nielsen class of $f_{i}^{n}, i=0,1$. If $N_{0}$ corresponds to $N_{1}$ via the homotopy $\mathrm{H}^{n}$, then

$$
I_{n}\left(f_{0}, N_{0}\right)=I_{n}\left(f_{1}, N_{1}\right) \text {. }
$$

Proof. For $i=0,1$, denote by $\alpha_{i}$ the lifting class of $f_{i}^{n}$ which represents $N_{i}$. Let $\tau \subset P(n)$. Let $L_{i, \tau}$ be the set of lifting classes $\beta$ of $f_{i}^{n: \tau}$ with $\beta^{n^{\prime}}=\alpha_{i}$, where $n^{\prime}=$ $n /(n: \tau)$ and $\beta^{n^{\prime}}$ is defined by $\beta^{n^{\prime}}=\left[h^{n^{\prime}}\right]$ for a lifting $h$ of $f_{i}^{n: \tau}$ with $\beta=[h]$. Then it is easy to see that Fix $\left(f_{i}^{n: \tau}\right) \cap N_{i}$ is a disjoint union of the Nielsen classes determined by all $\beta \in L_{i, r^{\prime}}$ and that there is a bijective correspondence between $L_{0, \tau}$ and $L_{1, \tau}$ via the homotopy $H^{n: \tau}$. Therefore, the result follows from the homotopy invariance theorem for the indices of Nielsen classes [11, Theorem 4.5, 3, Theorem 3, p. 94].

Remark. Proposition 2 can be generalized to compactly fixed maps on noncompact ENRs by using the generalized Nielsen fixed point theory on noncompact ENRs due to Fadell and Husseini [6].

We give two examples of the computation of $I_{n}(f, S)$ by using the homotopy invariance.

Example 1. If $f$ is homotopic to a map $\phi$ which has no periodic points, then by Proposition $2, I_{n}(f, N)=0$ for all $n$ and all Nielsen classes $N$ of $f^{n}$ (since for any homotopy $H$ from $f$ to $\phi, N$ corresponds to the empty set via $H^{n}$ ). In particular, if $f$ is homotopic to the identity map and the Euler characteristic of $M$ is zero, then the same conclusion holds. Therefore, in the special case where $f$ is a diffeomorphism of an annulus homotopic to the identity, Theorem 4 becomes a result of Kawakami [12], which are applied to the study of periodic systems of differential equations.

Example 2. We give an example where $I_{n}(f, S) \neq 0$. Let $M$ be a disk with $k$ open 
disks removed and $f: M \rightarrow M$ a continuous map. Assume that $f$ has a fixed point $x_{0}$. It is well known that the fundamental group $\pi=\pi_{1}\left(M, x_{0}\right)$ is a free group generated by $k$ generators $x_{1}, \ldots, x_{k}$. Let $B_{k}$ be the braid group on $k$ strings, $\sigma_{1}, \ldots, \sigma_{k-1}$ the generators of $B_{k}$, and $\rho: B_{k} \rightarrow$ Aut $(\pi)$ the homomorphism defined by $(\mathrm{cf},[1,(1.14)])$

$$
\begin{aligned}
\rho\left(\sigma_{i}\right)\left(x_{j}\right) & =x_{i} x_{i+1} x_{i}^{-1} & & \text { if } j=i, \\
& =x_{i} & & \text { if } j=i+1, \\
& =x_{j} & & \text { otherwise. }
\end{aligned}
$$

We assume that there exists $\sigma \in B_{k}$ such that $\rho(\sigma): \pi \rightarrow \pi$ coincides with the homomorphism $f_{*}: \pi \rightarrow \pi$ induced by $f$.

For an integer $i$, let $N_{i}$ denote the set of fixed points $x$ of $f^{n}$ such that $\mu\left(\left[c\left(f^{n} \circ c\right)^{-1}\right]\right)=i$, where $c$ is some path in $M$ from $x_{0}$ to $x$ and $\mu: \pi \rightarrow \mathbb{Z}$ is the homomorphism defined by $\mu\left(x_{i}\right)=1$. (Note that $c\left(f^{n} \circ c\right)^{-1}$ is a loop in $M$ based at $x_{0}$.) It is easy to show that $N_{i}$ is a union of Nielsen classes of $f^{n}$.

Let $\mathbb{Z}[t]$ be the ring of integer polynomials on the variable $t$ and its inverse. For a finite set $\tau$ of primes, define a homomorphism $\Psi_{\tau}: \mathbb{Z}[t] \rightarrow \mathbb{Z}[t]$ by $\Psi_{\tau}(t)=t^{d}$, where $d=\prod_{p \in \tau} p$. For $\sigma \in B_{k}$, let $B(\sigma)$ be the reduced Burau matrix of $\sigma[1$, p. 125]. Then $\operatorname{tr} B(\sigma)$ is an element of $\mathbb{Z}[t]$ and we have:

\section{Proposition 3.}

$$
\sum_{i \in \mathbf{Z}} I_{n}\left(f, N_{i}\right) \cdot t^{i}=-\sum_{\tau \in P(n)}(-1)^{|\tau|} \Psi_{\tau}\left(\operatorname{tr} B\left(\sigma^{n: \tau}\right)\right)
$$

Proof. Consider first the case of $n=1$. Let $\pi$ act itself on the right by $\gamma \xi=\xi^{-1} \gamma f_{*}(\xi)$, $\gamma, \xi \in \pi$, and let $R(f)$ denote the resulting set of orbits. There is a bijective correspondence $\Phi$ between $R(f)$ and the set of lifting classes of $f$ given by $\Phi([\gamma])=$ $[\gamma \circ \tilde{f}]$, where $\tilde{f}$ is a lifting of $f$ having a fixed point (such a lifting $\tilde{f}$ always exists) [11, Theorem 1.7]. For $\alpha \in R(f)$, let $N_{\alpha}$ denote the Nielsen class of $f$ determined by $\Phi(\alpha)$. Define an element $\hat{I}(f)$ of the free abelian group $\mathbb{Z}[R(f)]$ generated by the set $R(f)$ by

$$
\hat{I}(f)=\sum_{\alpha \in R(f)} I\left(f, N_{\alpha}\right) \cdot \alpha
$$

Then $\hat{I}(f)$ is a homotopy invariant; moreover, it is known that $\hat{I}(f)$ coincides with the generalized Lefschetz number $\hat{L}(f)$ defined by Husseini [10, Theorem (1.13)]. Also by [7, Theorem 2.3],

$$
\hat{L}(f)=1-\sum_{i=1}^{k} \Delta\left(\left(\partial \rho(\sigma)\left(x_{i}\right)\right) / \partial x_{i}\right),
$$

where $\partial / \partial x_{i}$ denotes the Fox derivative and $\Delta: \mathbb{Z}[\pi] \rightarrow \mathbb{Z}[R(f)]$ is the homomorphism induced by the natural projection. Define a homomorphism $\Gamma: \mathbb{Z}[R(f)] \rightarrow \mathbb{Z}[t]$ by $\Gamma\left(\left[x_{i}\right]\right)=t$. Then $\Gamma(\alpha)=t^{i}$ if and only if $N_{\alpha} \subset N_{i}$. Therefore by (5.3), (5.4),

$$
\sum_{i \in \mathbb{Z}} I\left(f, N_{i}\right) \cdot t^{i}=\Gamma(\hat{I}(f))=-\operatorname{tr} B(\sigma)
$$

and we obtain the desired equality in the case of $n=1$. (For another proof of (5.5), 
see [16, Proposition 2].) The equality for general $n$ can be proved by applying (5.5) to the maps $f^{m}, m \mid n$.

For example, assume $M$ has three holes and $\sigma=\sigma_{1} \sigma_{2}^{-1}$. Then,

$$
\operatorname{tr} B(\sigma)=1-t-t^{-1}, \operatorname{tr} B\left(\sigma^{2}\right)=1-2\left(t+t^{-1}\right)+t^{2}+t^{-2} \text {. }
$$

Therefore, by Proposition 3,

$$
\begin{aligned}
I_{2}\left(f, N_{i}\right) & =2 & & \text { if } i=1,-1, \\
& =-2 & & \text { if } i=2,-2, \\
& =0 & & \text { otherwise. }
\end{aligned}
$$

The maps treated in Example 2 appear naturally in the theory of periodic systems (see [16, Lemma 2]). Hence Theorem 4 and Proposition 3 can be applied to the study of such systems.

\section{REFERENCES}

[1] J. S. Birman. Braids, links, and mapping class groups. Ann. Math. Studies 82 (Princeton University Press: Princeton, 1974).

[2] F. E. Browder. The Lefschetz fixed point theorem and asymptotic fixed point theorems. Lecture Notes in Math. 446. Springer-Verlag: Berlin, 1975), pp. 96-122.

[3] R. Brown. The Lefschetz Fixed Point Theorem (Scott-Foresman: Chicago, 1971).

[4] A. Dold. Fixed point index and fixed point theorem for Euclidean neighborhood retracts. Topology 4 (1965), 1-8.

[5] A. Dold. Fixed point indices of iterated maps. Invent. Math. 74 (1983), 419-435.

[6] E. Fadell \& S. Husseini. Local fixed point index theory for non-simply-connected manifolds. Illinois J. Math. 25 (1981), 673-699.

[7] E. Fadell \& S. Husseini. The Nielsen number on surfaces, Contemp. Math. 21. Amer. Math. Soc. (1983), pp. 59-98.

[8] J. Franks. Some smooth maps with infinitely many hyperbolic periodic points. Trans. Amer. Math. Soc. 226 (1977), 175-179.

[9] J. Franks. Period doubling and the Lefschetz formula. Trans. Amer. Math. Soc. 287 (1985), 275-283.

[10] S. Husseini. Generalized Lefschetz numbers. Trans. Amer. Math. Soc. 272 (1982), 247-274.

[11] B. Jiang. Lectures on Nielsen fixed point theory. Contemp. Math. 14. Amer. Math. Soc. (1983).

[12] H. Kawakami. Qualitative properties of forced oscillations on the cylindrical phase surface (Japanese). Trans. IECE Japan 64-A (1981), 916-923.

[13] M. A. Krasnosel'skii and P. P. Zabreiko. Geometrical Methods of Nonlinear Analysis. Grundlehren der mathematischen Wissenschaften 263 (Springer-Verlag: Berlin, 1984).

[14] N. Levinson. Transformation theory of non-linear differential equations of the second order. Ann. Math. 45 (1944), 723-737, Corrections. ibid. 49 (1948), 738.

[15] J. L. Massera. The number of subharmonic solutions of non-linear differential equations of the second order. Ann. of Math. 50 (1949), 118-126.

[16] T. Matsuoka. The number and linking of periodic solutions of periodic systems. Invent. Math. 70 (1983), 319-340.

[17] H. O. Peitgen. On the Lefschetz number for iterates of continuous mappings. Proc. Amer. Math. Soc. 54 (1976), 441-444.

[18] K. Shiraiwa. A generalization of the Levinson-Massera's equalities. Nagoya Math. J. 67 (1977), 121-138.

[19] P. P. Zabreiko and M. A. Krasnosel'skii. The rotation of vector fields with superpositions and iterations of operators (Russian). Vestnik Jaroslav. Univ. Vyp. 12 (1975), 23-37. 